\title{
Los programas de infoentretenimiento como sustituto de los debates electorales: el caso de Miguel Ángel Revilla
}

\section{Infotainment programs as substitutes for leaders' debates: the case of Miguel Ángel Revilla}

Noelia Fontecoba. Universidad de Vigo. España.

noeliafontecoba@gmail.com

[CV]

Ana Belén Fernández-Souto. Universidad de Vigo. España.

abfsouto@uvigo.es

$[\mathrm{CV}]$ (1) $\mathrm{G}$

Iván Puentes-Rivera. Universidad de A Coruña. España.

i.puentes@udc.es

$[\mathrm{CV}](\mathrm{C}$

Este artículo forma parte de los trabajos desarrollados en el marco del proyecto de investigación "DEBATv, Debates Electorales Televisados en España: Modelos, Proceso, Diagnostico y Propuesta" (CSO2017-83159$R$ ), proyecto de $I+D+I$ (Retos) financiado por el Ministerio de Innovación, Ciencia y Universidades y la Agencia Estatal de Investigación (AEI) del Gobierno de España, con el apoyo del Fondo Europeo de Desarrollo Regional (FEDER) de la Unión Europea (UE).

Cómo citar este artículo / Referencia normalizada

Fontecoba, N., Fernández-Souto, A. y Puentes-Rivera, I. (2020). Los programas de infoentretenimiento como sustituto de los debates electorales: el caso de Miguel Ángel Revilla. Revista Latina de Comunicación Social, (76), 59-80.

https://www.doi.org/10.4185/RLCS-2020-1437

\section{RESUMEN}

En la actualidad, el papel del líder político ha cobrado una nueva dimensión. La televisión y las redes sociales han hecho que, a través de la imagen, la atención se concrete en el candidato y no en ideas o programas. De esta forma, el líder lo es no sólo por sus cualidades intelectuales, sino también por su capacidad mediática. Con este nuevo panorama, donde se realiza una política más icónica y de espectáculo, la televisión ha incorporado en sus programas de infoentretenimiento la presencia de políticos con el fin de atraer el mayor número de audiencia posible. Como consecuencia, los ciudadanos opinan sobre los gestos, el tono o la vestimenta de los candidatos. Este contexto hace que las estrategias de comunicación cambien y que la comunicación no verbal sea uno de los aspectos más estudiados en los gabinetes de comunicación. Por este motivo, en este estudio trabajo se analiza la comunicación no verbal de Miguel Ángel Revilla en cuatro programas de infoentretenimiento.

PALABRAS CLAVE: comunicación no verbal; comunicación política; infoentretenimiento; Miguel Ángel Revilla. 


\begin{abstract}
Currently, the role of political leaders has acquired a new dimension. Television and social media have made, through image, the attention be focused on the candidate and not on ideas or programs. Thus, the leader is not just as such for his/her intellectual qualities, but also for his/her media competence. With this new picture, where more iconic and show centered politics take place, television has incorporated the presence of politicians in their infotainment programs with aims of drawing the highest number of audience possible. As a consequence, citizens voice about the candidates' gestures, tone and clothes they wear. This context makes communication strategies change and nonverbal communication be one of the most studied aspects by press service. For this reason, the nonverbal communication of Miguel Ángel Revilla on four infotainment programs is analyzed in this study.
\end{abstract}

KEYWORDS: nonverbal communication; political communication; infotainment; Miguel Ángel Revilla.

\title{
CONTENIDOS
}

1. Introducción. 2. Marco teórico. 2. Debates electorales y fact-ckecking. 2.1. La comunicación no verbal. 2.2. La comunicación política. 2.2.1. La televisión como escenario principal de la comunicación política. El fenómeno de la videopolítica. 2.2.2. La americanización y personalización de la política. 2.3. El infoentretenimiento político televisivo. 2.3.1. Infoentretenimiento televisivo y política. 2.3.2. Formatos del infoentretenimiento político televisado. Tipología y características. 3. Método. 3.1. Objetivos. 3.2. Hipótesis. 3.3. Metodología. 4. Resultados. La imagen política y mediática de Miguel Ángel Revilla. 4.1. Análisis de la comunicación no verbal de Miguel Ángel Revilla en programas de infoentretenimiento. 4.1.1. Espejo Público. 4.1.2. Mi casa es la tuya. 4.1.3. Chester in Love. 4.1.4. La Sexta Noche. 4.2. Resultados de la investigación: Comparación de resultados de los cuatro programas analizados: Espejo Público, Mi casa es la tuya, Chester in Love y La Sexta Noche. 5. Conclusiones. 6. Referencias bibliográficas.

\section{Introducción}

El papel del líder ha marcado la política desde antiguo, sin embargo, es ahora, en la actualidad, cuando cobra más fuerza. Este cambio es visible en los primeros estudios electorales que datan de los años 40. Sin embargo, la aparición de la televisión y la retransmisión de los debates electorales a través de este medio, marcan un punto de inflexión en la historia de la comunicación política, donde el líder político cobra un gran protagonismo.

En este contexto, a partir de los años 90, se produce un gran aumento de la presencia de los líderes políticos en los programas de infoentretenimiento televisivos. Así, los candidatos aparecen jugando al baloncesto, bailando o enseñando sus casas en este tipo de programas. De esta manera, las televisiones alcanzan grandes números de audiencia y los políticos consiguen entrar en los salones de los votantes.

Esta personalización de la política, que tiene su máxima representación en los programas de infoentretenimiento televisivos, ha desencadenado que los ciudadanos juzguen los resultados de las intervenciones de los políticos por sus expresiones, su tono de voz, su vestimenta y otro sinfín de aspectos. Por lo tanto, la comunicación no verbal ha cobrado una gran importancia y es examinada por la mayoría de la población. De esta forma, los gabinetes de comunicación y asesores de imagen estudian minuciosamente cada movimiento e intentan aconsejar a los líderes políticos sobre cuáles son las mejores poses, los gestos más apropiados o el tono más recomendado para cada ocasión. 
Dada la importancia que recibe la comunicación no verbal sobre los líderes políticos, se decide analizar la comunicación no verbal del Presidente de Cantabria, Miguel Ángel Revilla. Para ello nos valdremos del visionado de cuatro programas de infoentretenimiento emitidos en España. Dos de ellos del año 2017 (Chester in Love y Mi casa es la tuya) y otros dos de este mismo año, 2019 (Espejo Público y La Sexta Noche).

\section{Marco teórico}

\subsection{La comunicación no verbal}

Según Cestero (2006) no es hasta la segunda mitad del siglo XX cuando nace la comunicación no verbal como disciplina. Más tarde, muchos fueron los psicólogos, investigadores y autores que trataron de demostrar su importancia. Uno de los primeros fue el psicólogo Albert Mehrabian (como se citó en Alonso, 2017, p. 6) al averiguar en 1971 que, en el acto de comunicación, el sentimiento del hablante se transmite al interlocutor por medio de una combinación de lenguaje fónico (solo palabras) en un 7\%, un $55 \%$ mediante el lenguaje gestual y un 38\% a través del tono de voz, los matices y otros sonidos.

Años después Pease (2011) menciona que el impacto que los mensajes provocan sobre quienes escuchan proviene entre un $7 \%$ y un $10 \%$, de las palabras, entre un $20 \%$ y un $30 \%$ del paralenguaje $\mathrm{y}$, entre un $60 \%$ y un $80 \%$ de la quinésica o lenguaje corporal.

Poyatos (2003, p. 68) añade en su definición la importancia de la cultura para comprender este fenómeno y es que para él la comunicación no verbal son "las emisiones de signos activos o pasivos, constituyan o no comportamiento, a través de los sistemas no léxicos somáticos, objetuales y ambientales contenidos en una cultura, individualmente o en mutua coestructuración".

Por otra parte, otros autores como Flora Davis conciben la comunicación no verbal como parte de la comunicación verbal (Davis, 2010, p.7).

Aglutinando a todos ellos, Cabana incide en que

La comunicación no verbal es una forma de interacción silenciosa, espontánea, sincera y sin

rodeos. Ilustra la verdad de las palabras pronunciadas al ser todos nuestros gestos un reflejo

instintivo de nuestras reacciones que componen nuestra actitud mediante el envío de mensajes

corporales continuos. De esta manera, nuestra envoltura carnal desvela con transparencia

nuestras verdaderas pulsiones, emociones y sentimientos. Resulta que varios de nuestros gestos

constituyen una forma de declaración silenciosa que tiene por objeto dar a conocer nuestras

verdaderas intenciones a través de nuestras actitudes. (Cabana, 2008, p. 21)

Así, está aceptado que dentro de la comunicación no verbal se diferencian los siguientes sistemas:

1. El sistema paralingüístico. Es aquello que está más allá de las palabras (para-lenguaje). Estas son las ocho formas de las cualidades primarias basada en las aportaciones de Poyatos (1994): timbre, tono, resonancia, intensidad o volumen, tempo o velocidad, entonación, ritmo y duración silábica. 
2. El sistema kinésico. Para Poyatos (como se citó en Moyá, 2016, p. 86) la kinesia son: "los movimientos y posiciones de base psicomuscular conscientes o inconscientes que poseen valor comunicativo intencionado o no". Según él (1994) se distinguen tres categorías quinésicas: Los gestos, las maneras o formas convencionales de realizar acciones o movimientos y las posturas o posiciones estáticas comunicativas: Los gestos (faciales y corporales), las maneras y las posturas.

3. El sistema proxémico. Forner (1987) define la proxémica como las relaciones que se establecen o determinan entre los interactuantes según las distancias que se sitúan entre ellos y también respecto a los objetos. Estas distancias tienen normas y reglas explícitas o implícitas en función de las situaciones, los ambientes y las culturas. Hall (1973) distingue entre Distancia íntima (entre 15 y 45 centímetros), personal (entre 46 y 120 centímetros), social (entre 120 y 360 centímetros) y pública (más de 360 ).

4. El sistema cronémico. El tiempo también comunica, bien pasivamente, ofreciendo información cultural, bien activamente, modificando o reforzando el significado de los elementos del resto de sistemas de comunicación humana. Su estudio se ha denominado cronémica, que se define como la concepción, la estructuración y el uso que hace del tiempo el ser humano (Cestero, 2006, p. 64).

\subsection{La comunicación política}

Para Trent y Friedenberg (como se citó en Canel, 2005, p. 20) la comunicación política es el conjunto de símbolos creados por los medios de comunicación para que la sociedad mantenga la consciencia de la existencia de unas instituciones políticas; es decir, son las imágenes las que ayudan a que el pueblo recuerde que se vota, que se controla al gobierno o que la constitución funciona. Estos autores ponen el foco en los medios de comunicación. Para ellos, los medios son los responsables de crear símbolos con el fin de que el pueblo recuerde la existencia de las instituciones políticas.

Frente a esta definición está la de D. Nimmo (como se citó en Monzon, 2006, p. 93) quien afirma que "una comunicación (actividad) puede ser considerada política en virtud de las consecuencias (actuales y potenciales) que regulan la conducta humana bajo ciertas condiciones de conflicto". Según él podemos hablar de comunicación política cuando exista interacción de opiniones sobre los asuntos que interesan al gobierno y a los ciudadanos, de otra forma solo será comunicación entre individuos.

\subsubsection{La televisión como escenario principal de la comunicación política. El fenómeno de la videopolítica}

Quizás los primeros en percatarse de las ventajas que ofrece la televisión fueron Roosevelt y Landon en el año 1936 en Estados Unidos. Estos candidatos utilizaron la televisión para hacer campaña. Sin embargo, como explica Caamaño $(2016$, p. 9) "se trató de un acontecimiento experimental al que muy poca población tuvo acceso, por lo que no puede ser considerado como modelo de comunicación política, sino más bien como un antecedente inmediato de ésta".

Hubo que esperar, según Martínez (2006), hasta mediados de la década de los 70 para realizar una de las primeras investigaciones sobre la importancia de la televisión en relación a la política y la comunicación. El encargado de poner este hecho en la palestra fue Roland Cayrol que tras sus estudios proporcionó un resultado que en aquel momento sorprendió al mundo académico: la televisión constituía el medio de masas preferido por los votantes. Y es que la televisión supuso un cambio muy importante a la hora de realizar comunicación política. Así lo afirma Sanmartí (como se citó en Caamaño, 2016). 
Además, los debates son uno de los acontecimientos más seguidos por la audiencia desde épocas pasadas. El interés por este formato dura hasta la actualidad, así lo demuestran las cifras: según Barlovento Comunicación (2019) 9.477.000 espectadores siguieron el último debate político retransmitido el 23 de abril de 2019 y consiguió un 48,7\% de cuota de pantalla.

Por otra parte, hay que apuntar que con la aparición de la televisión, el lenguaje político se ha visto "obligado" a adaptarse a este medio. Según Pérez (2003, p. 29): "el lenguaje político se ha visto influido por un hecho indudable: la sociedad del conocimiento es hoy decididamente audiovisual". Aparece así la importancia de la imagen y el vídeo como elementos determinantes en la comunicación política.

En consonancia con esta primacía de la imagen y el vídeo varios autores consideran necesario poner en escena el término videopolítica. Muchos autores le han dado una definición a esta herramienta: Para Carabajal (2011, p.2), "la videopolítica es un fenómeno que alude a la superficie sobre la cual se ve reflejada la política".

En cambio, Fischer (2005) dirige el interés en el lado publicitario: "La videopolítica es la manera de hacer política mediatizada, principalmente, por la imagen televisiva, en donde las empresas de publicidad dirigen las agendas de muchos temas ciudadanos y de gobierno, creando imágenes atrayentes que venden a los electores como publicidad" (p. 133).

Para Sartori (2003) la videopolítica se refiere solo a uno de los muchos aspectos del poder del vídeo, que pone el foco en los procesos políticos y nos muestra como "ser políticos" y cómo "gestionar la política".

Para finalizar debemos añadir que la videopolítica y la televisión se están transformando. Debido al auge de las nuevas tecnologías, Internet y las redes sociales la forma de consumir la televisión es diferente y las posibilidades que ofrece mucho mayores. Así lo afirma Kantar Media (2019, p. 4): "La televisión es ahora una fusión flexible de experiencias visuales y de sonido que se ofrece en varios dispositivos. Se puede planificar la visualización y los programas se pueden ver en cualquier momento, lugar y a la carta".

A pesar del auge de las redes sociales no nos debemos olvidar de que la televisión sigue ocupando un espacio importante. Así lo certifica AIMC (2019) en su estudio Marco General de los Medios en España 2019 donde se indica que el consumo diario de televisión por parte de los españoles en el año 2018 fue de 210,3 minutos seguido por Internet con 139,8 minutos. Gracias a estos datos podemos decir que al no perder la televisión su reinado la política seguirá usando este medio para comunicarse y poder llegar al máximo número de telespectadores, sin obviar, evidentemente, la importancia creciente de las redes sociales, en la comunicación política en general y la videopolítica en particular, trascendentales en la estrategia comunicativa de multitud de acontecimientos públicos extranjeros y nacionales (Cartes Barroso, 2018).

\subsubsection{La americanización y personalización de la política}

Para conseguir una comprensión más detallada de la centralidad de la televisión en la comunicación política, es imprescindible atender al proceso denominado "americanización".

Los primeros en plantear este término fueron Swanson y Mancini. Así lo corrobora Orejuela (2006, p. 57): "Fueron Swanson y Mancini los que plantearon en 1996 la hipótesis de la americanización, que les sirvió para constatar cómo candidatos y partidos, de diversas culturas, toman como modelo a los Estados Unidos". 
$\mathrm{Y}$ es que Estados Unidos funciona como una especie de "laboratorio social" donde no sólo se practica con la comunicación política sino también con tendencias culturales que posteriormente se adoptan en los diferentes países. Esta adopción y seguimiento de los modelos de campañas electorales que llegaban desde Estados Unidos llevaron a los autores anteriormente mencionados al término "americanización", aplicado hoy por otros muchos, también, a la comunicación política, al menos de partido, dominante en España (Padilla Castillo, 2019), al igual que ocurre con la espectacularización de los debates electorales desde hace años, copiando el modelo americano (Padilla Castillo, 2015) en busca de una mayor influencia y repercusión de los mismos (Gallego Reguera y Bernárdez Rodal, 2017) o la complementariedad entre cine y comunicación política (Rodríguez Vidales y Padilla Castillo, 2018).

El desarrollo que la televisión ha tenido en las últimas décadas ha favorecido un marketing político más centrado en subrayar las cualidades personales de quien encarna la política, del candidato, en detrimento de las características técnicas y abstractas del partido o del programa. Es éste el fenómeno de la "personificación de la política" que ha contribuido al encumbramiento de los líderes en perjuicio del partido como institución" (Canel, 2005, p. 50).

Además, debemos reflexionar sobre las nuevas estrategias de los medios por noticias de cuestiones personales de los líderes políticos. Si nos fijamos cada vez es más difícil encontrar una noticia de política sin contenido personal del líder, de la misma forma que resulta casi imposible asistir a una conversación sobre política que no trate temas personales de los líderes de los partidos.

Para finalizar debemos apuntar que esta personalización de la política tiene unas consecuencias. Según Laguna (2011) son en primer lugar que los ciudadanos juzguen los resultados de las intervenciones de los políticos por sus expresiones, sus miradas y otros aspectos personales.

En segundo lugar, el debilitamiento de las identidades partidarias como referencia clave de la decisión electoral, lo que además supone una desideologización de la sociedad al sustituirse la identidad partidaria por temas difundidos por los medios de comunicación.

Y recoge como tercera consecuencia las palabras de Bouza que explica que esta nueva dimensión implica asumir que son los medios los responsables últimos de la comunicación política, es decir, que el resultado final de la comunicación política pasa a depender de la capacidad de visibilidad que logre el líder en los medios y de la fuerza que tenga la imagen que proyecte.

\subsection{El infoentretenimiento político televisivo}

Desde los inicios de la televisión ésta ha sido empleada con diversos fines, pero sin duda uno de los más utilizados es el entretenimiento. De esta forma aparece el fenómeno del infoentretenimiento. Así lo afirma Krüger (como se citó en García, 2007, p. 50) “Al menos desde 1988, el término anglosajón infotainment se utiliza para designar a determinados programas que combinan la información con el entretenimiento".

Según este autor los inicios del infoentretenimiento surgen a finales de los 80 y principios de los 90. Sin embargo, para Starck (1997) la existencia de este fenómeno se produce a finales de la década de los 60 y principios de los 70. Además, lo sitúa en los canales locales de Estados Unidos.

Ortells (2012, p. 100) añade los temas que son más atractivos para el fenómeno: "El infoentretenimiento se ha consolidado como un fenómeno periodístico que aúna en un mismo espacio rasgos propios de los programas informativos tradicionales con características inherentes al 
género del entretenimiento. Se implanta así una lógica informativa en la que los temas más suaves, con enfoques humanos, prevalecen por encima de aquellas temáticas vinculadas a las cuestiones más estrictamente conectadas al interés público.

Berrocal, Redondo, Martín y Campos (2014) incorporan el sensacionalismo como elemento presente en el infoentretenimiento. De esta forma, y siguiendo a estos autores el infoentretenimiento se produce en un escenario de espectacularización y sensacionalismo donde la imagen, el show, y la personalización tienen protagonismo. De hecho, Berrocal et al. (2014) afirman: "Dentro de esa estrategia, se incardina la espectacularización de la realidad que da lugar al infoentretenimiento. Un fenómeno que enlaza con el sensacionalismo propio de la prensa popular pero que adquiere nuevas características según "se va extendiendo y adaptando al resto de medios de comunicación (p.86). Además, Oliva y Sitjà (2007) también explican que el infotainment es visible en un espacio donde confluyen las características de lo audiovisual y añade lo narrativo de forma que la información está ligada al entretenimiento a través de técnicas relacionadas con la imagen.

\subsubsection{Infoentretenimiento televisivo y política}

Dentro de los programas de infoentretenimiento televisivo uno de los temas más recurridos es la política. De esta forma, las noticias políticas son tratadas de una forma menos seria. Así lo recoge Berrocal et al. (2014, p. 89): "Pero, al mismo tiempo, el infoentretenimiento se caracteriza por recoger aquellas informaciones denominadas serias (política, economía) tratándolas de una manera dramática, paródica o humorística". Además, añaden: "La información política, en este caso, no recibe un tratamiento riguroso como el que se asocia de manera tradicional a este tipo de contenidos, sino que se transmite con la finalidad de entretener buscando la anécdota o la burla de forma que resulten piezas más atrayentes para el público".

Berrocal y Cebrián (2009, p. 47) también están de acuerdo con los autores mencionados anteriormente y agregan: "El problema que hoy atisbamos es que la información política televisada está copiando un formato que tiene mucho que ver con: la espectacularización, la conflictividad, o lo trivial y anecdótico".

De este modo y como explican estos autores el tratamiento que ahora reciben los temas políticos está experimentando una serie de cambios. De hecho, Ortells (2012, p. 99) reconoce que este fenómeno ha producido interés en los investigadores y que: "Todos coinciden en señalar que la televisión es el medio más utilizado para consumir información política, y en que, efectivamente, se están produciendo variaciones en la producción y consumo de contenidos informativos”.

Para poder comprobar esto no hay más que ver cómo los políticos y políticas aparecen en los programas televisivos de infoentretenimiento jugando al baloncesto, bailando o enseñando sus casas.

Y es que como afirma Berrocal (2015, p. 4): “Esta tendencia, que concede a la información política una dosis de superficialidad, se amplía a partir de los años 90 con la presencia de los políticos en programas magazines o "contenedores", donde comparten espacio con personajes del mundo del corazón o concursantes de los últimos programas reality show de la cadena, y también con su presencia figurada o no en programas de parodia o sátira política".

El hecho de que la televisión unifique todas las variables del discurso político y las convierta en espectáculo, esparcimiento o entretenimiento, ha provocado que lo anecdótico ocupe el primer plano en aras de conseguir réditos electorales a través de esa humanización del candidato (Alonso p.91). 
Durante las campañas electorales este fenómeno es más visible todavía ya que los candidatos políticos acuden a una gran cantidad de programas de este tipo con la intención de acercar al telespectador y a sus posibles votantes transmitiendo una imagen más natural y cercana. De esta forma asegura Valencia $(2015$, p. 27) "Esta relación entre política e imagen cambia la imagen y el discurso de los políticos, las campañas electorales y, sobre todo, eleva a los gabinetes de comunicación y a los asesores de imagen a un papel mucho más relevante”.

Por ello, los propios políticos tratando de acercarse al, cada vez, más amplio colectivo que se encuentra en la Red, han sumado estrategias de comunicación dirigidas a este nuevo medio. Esta labor emprendida por la fuente política para aproximarse al ciudadano que accede a la Red, se ve acompañada por la propia reacción de los internautas, que acuden a Internet en busca de su "propia" información política (Berrocal, Campos y Redondo, 2012, p. 108).

Como conclusión debemos apuntar que los programas de infoentretenimiento con contenido político son habituales en la parrilla televisiva española y que este fenómeno se está expandiendo cada vez más a otros medios como por ejemplo Internet. Ahora que hemos analizado esta situación creemos relevante analizar los tipos de formatos de infoentretenimiento político existentes con el fin de segmentarlos y aproximarnos más al tema que nos ocupa.

\subsubsection{Formatos del infoentretenimiento político televisado. Tipología y características}

En la actualidad son muchos los programas de infoentretenimiento que se emiten en televisión, por este motivo es preciso agruparlos y analizar sus particularidades.

Berrocal y Cebrián realizan la siguiente clasificación de formatos de infoentretenimiento político televisado:

a. Formatos políticos espectacularizados: programas que tienen una estructura tradicional de un programa político donde podemos ver a especialistas comentando los asuntos de actualidad con técnicas específicas del entretenimiento con el objetivo de divertir a los telespectadores.

b. Magazines politizados: magazines tradicionales en los que se hace un hueco a los asuntos políticos. El factor de espectacularización en este caso reside precisamente en el hecho de incluir lo político en un marco que le es ajeno (Berrocal y Cebrián, 2009, p.12).

c. Infoshows políticos: Se trata de un formato claramente orientado al ocio, pero que espectaculariza lo político en dos sentidos: por un lado maneja paradójicamente la actualidad política ironizando sobre ella a la vez que convirtiéndola en el centro del programa, y por otro implica a los políticos en actividades muy alejadas de la de sus funciones clásicas (Berrocal y Cebrián, 2009, p. 13).

\section{Método}

\subsection{Objetivos}

Objetivos principales:

1. Averiguar cuál es el gesto más repetido por Miguel Ángel Revilla en los cuatro programas de infoentretenimiento televisivo a analizar.

2. Descubrir qué transmite su gesto más repetido.

3. Comprobar si sus gestos se corresponden con un discurso creíble.

4. Comparar la comunicación no verbal de Miguel Ángel Revilla en los programas de infoentretenimiento televisivos del año 2017 al 2019. 
Objetivos secundarios:

5. Detectar si existe una influencia del entorno en la indumentaria que utiliza Miguel Ángel Revilla durante los cuatro programas seleccionados.

\subsection{Hipótesis}

- Hipótesis I: El Presidente de Cantabria, a través de su comunicación no verbal, se muestra seguro de sí mismo en sus intervenciones en programas de infoentretenimiento televisivos.

- Hipótesis II: En las entrevistas concedidas después de ganar las elecciones autonóminas de este mismo año, Miguel Ángel Revilla muestra a través de sus gestos más seguridad y alegría que en las intervenciones del 2019 donde ya ha ganado por mayoría las elecciones autonómicas y conseguido un diputado en las elecciones generales.

- Hipótesis III: Miguel Ángel Revilla emplea una vestimenta más informal cuando se encuentra en su tierra, Cantabria, hecho que le aporta una imagen más cercana y campechana.

- Hipótesis IV: El Presidente de Cantabria no pone ningún inconveniente en contestar preguntas sobre cuestiones de su vida privada.

\subsection{Metodología}

Además de realizar un intenso vaciado bibliográfico, para realizar el marco teórico de la investigación, este trabajo se completa con el visionado y análisis tanto cuantitativo como cualitativo de cuatro programas de infoentretenimiento: Mi casa es la tuya (Telecinco) y Chester in Love (Cuatro) emitidos en el año 2017 y Espejo Público (Antena 3) y La Sexta Noche (La Sexta) del año 2019.

La elección de estos programas, se debe en un primer lugar, a que se pretende realizar un análisis de la comunicación no verbal de Miguel Ángel Revilla en programas de diferentes cadenas de televisión españolas. Se encontraron varios programas más que podrían encajar en el objeto de estudio, pero han sido obviados debido a que la intervención del Sr. Revilla no excedía los 15 minutos.

La selección es la siguiente:

- Mi casa es la tuya y Chester in Love, que datan del año 2017 y en los que el Presidente de Cantabria interviene más de 15 minutos.

- La Sexta Noche, del 2019, se elige por ser la primera entrevista que Miguel Ángel Revilla concede después de que el Partido Regionalista de Cantabria gane por primera vez en la historia un diputado en las elecciones generales.

- Algo parecido sucede con el programa Espejo Público, se selecciona por ser la primera entrevista al Presidente de Cantabria después de ganar con mayoría de votos las elecciones autonómicas de este mismo año.

Tras la elección de estos programas y para realizar el correspondiente análisis, se elabora una plantilla ad hoc donde se recogen los principales signos del comportamiento no verbal del Sr. Revilla. A partir de los datos extraídos del visionado de los programas se han obtenido unos resultados por cada programa y se han extrapolado unas conclusiones comparativas entre ellos.

\section{Resultados. La imagen política y mediática de Miguel Ángel Revilla}

Además de su faceta política, el presidente cántabro se ha convertido en uno de los rostros televisivos más conocidos, gracias a su presencia en muchas tertulias televisivas. 
En base a ellas, podemos afirmar que Miguel Ángel Revilla produce una atracción innata sobre los medios de comunicación y, en concreto, sobre los programas de infoentretenimiento. Este interés de los medios por entrevistar al presidente puede deberse a que Revilla ofrece justo lo que demandan este tipo de programas: información y entretenimiento. El presidente se muestra natural en cada intervención y ha protagonizado momentos estelares como por ejemplo como recoge Antena 3 (2017) cambiar la letra del himno de Cantabria y cantarlo junto con el presentador Pablo Motos en el programa El Hormiguero.

Más recientemente y como explica Garrido (2019) el presidente cántabro acudió al Concurso de Ordeño de Trasierra en el municipio cántabro de Ruiloba el pasado mes de febrero donde bebió leche recién ordeñada. Gran cantidad de medios se hicieron eco de la noticia.

Pero el presidente no sólo es popular en la televisión, sino que cuenta con 326.000 seguidores en su cuenta de Instagram (a día de hoy, 5 de mayo de 2019). Además, es el segundo político con más número de seguidores en las redes sociales, sólo lo supera el líder de Podemos, Pablo Iglesias. Además de su fuerte presencia en las redes sociales, EuropaPress apunta que, según el estudio realizado por la plataforma Dyntra, Miguel Ángel Revilla es el presidente autonómico con mejor índice de transparencia: En la actualidad el presidente cántabro afronta con 76 años su cuarta legislatura como Presidente de Cantabria.

\subsection{Análisis de la comunicación no verbal de Miguel Ángel Revilla en programas de infoentretenimiento}

\subsubsection{Espejo Público}

Espejo Público es un programa matinal presentado por la periodista Susanna Griso en la cadena Antena 3. En este caso, el programa analizado data del día 29 de mayo de 2019, dura un total de 3 horas 46 minutos y 37 segundos y la entrevista que se realiza a Miguel Ángel Revilla se produce cuando el programa lleva 1 hora, 15 minutos y 17 segundos emitidos, durando un total de 29 minutos y 12 segundos.

Las características en las que se produce esta entrevista son diferentes a las habituales ya que se realiza en un taxi. La presentadora Susanna Griso se desplaza en taxi junto a Miguel Ángel Revilla hasta el aeropuerto Adolfo Suárez Madrid-Barajas, allí mantienen la primera entrevista que concede Miguel Ángel Revilla después de ganar las elecciones autonómicas de Cantabria 2019. El presidente apura sus últimas horas en Madrid para ofrecer su primera entrevista después de haber ganado las elecciones autonómicas de este mismo año. En el taxi Susanna Griso y Revilla charlan sobre el nuevo panorama político.

El presidente se presenta vestido con un traje azul marino, camisa blanca y corbata con rayas finas. Este traje lo acompaña con unos zapatos negros de cordón. El Presidente de Cantabria concede esta entrevista vestido de una forma elegante, mostrando a través de su indumentaria que posee un cargo importante.

Una vez que comienza la entrevista Susanna Griso y Miguel Ángel Revilla se sientan en los asientos traseros del taxi. Miguel Ángel Revilla presenta una postura relajada con la espalda apoyada en el respaldo y las piernas cruzadas durante todo el trayecto.

El movimiento de los ojos, su mirada, suele mantenerse firme a la presentadora, mirándola a los ojos. Además, es una mirada franca que muestra la seguridad que tiene en sí mismo y en su diálogo. Estos gestos se intercalan con una mirada al frente para ver en qué calle de Madrid se encuentran, pero en ningún caso muestra signos de estar distraído o inseguro. 
En cuanto a la sonrisa, se muestra con una sonrisa auténtica durante toda la entrevista, algo que coincide con su actitud y su rostro. Se aprecia alegría y tranquilidad.

En relación a sus movimientos con las manos hace un total de 43 gestos. El más repetido es la palma de las manos hacia arriba con 13 veces, algo que indica que es franco y honesto con sus palabras.

En segundo lugar, se encuentra el gesto que hemos denominado "Dedos índice y pulgar unidos y movimiento de arriba abajo", que lo utiliza 6 veces para reafirmar sus palabras, para recalcar y fijar los conceptos que estaba pronunciando ya que su diálogo y sus gestos hablan de la misma forma. También emplea 5 veces el dedo índice apuntando hacia arriba con la misma finalidad.

Por otra parte, sólo utiliza una vez la postura de manos en los bolsillos. Emplea este gesto al entrar en el aeropuerto y ante la pregunta de Susanna Griso sobre su futura sucesión en la presidencia regional. En ese momento Miguel Ángel Revilla camina despacio con las manos en los bolsillos y se nota incómodo con la pregunta. Miguel Ángel Revilla emplea un total de 4 veces el gesto de representación del yo, lo que indica que habla bastante sobre sí mismo.

En cuanto a las piernas, al realizarse la entrevista en un taxi la mayoría del tiempo está sentado y con las piernas cruzadas, salvo cuando salen del taxi para entrar en el aeropuerto. Que el Presidente de Cantabria aparezca con las piernas cruzadas en esta ocasión se puede deber a una postura que se obtiene para estar más cómodo ya que el asiento delantero del taxi está muy próximo. Al contrario de lo que puede indicar esta postura (actitud negativa, defensiva) considero que en este caso Miguel Ángel Revilla lo emplea como un gesto de comodidad y relajación.

En su conjunto, Miguel Ángel Revilla muestra una actitud tranquila y a la vez segura y firme. Acompaña sus movimientos con gestos que reafirman su discurso y al mismo tiempo se encuentra disfrutando de la entrevista con una sonrisa auténtica y sin mostrar signos de enfado o incomodidad.

Todos estos gestos están acompañados de un timbre medio-alto que emplea normalmente. A diferencia de otras intervenciones el tempo que emplea es lento y su ritmo suave con lo que demuestra su tranquilidad.

\subsubsection{Mi casa es la tuya}

Mi casa es la tuya es un programa presentado por Bertín Osborne en la cadena Telecinco. En concreto el programa se emite los viernes a las 22:00 horas. Anteriormente este programa se denominaba En la tuya o en la mía. Como indica su nombre, el programa se basa en realizar entrevistas a personajes famosos en sus casas.

En concreto el programa a analizar se emitió el día 16 de marzo de 2017 a las 22 horas, tiene una duración de 1 hora 35 minutos y 57 segundos y la entrevista se realiza en varias localizaciones. En un principio se grava en un mirador situado en Cosgaya, un pueblo de Cantabria. A continuación, la propia entrevista tiene lugar en una casa con ubicación exacta desconocida, pero si sabemos que está situada en Cantabria. Finalmente, Miguel Ángel Revilla y Bertín Osborne visitan el Monasterio de Santo Toribio de Liébana situado en Camaleño, otro pueblo cántabro. Por último, Miguel Ángel Revilla y Bertín Osborne comen con Aurora Díaz, la mujer de Miguel Ángel Revilla en un restaurante con vistas a los Picos de Europa.

Miguel Ángel Revilla aparece con chaqueta de color negro y abrochada. Utiliza esta prenda en las zonas que están al aire libre. Por debajo viste una camisa azul claro y unos pantalones vaqueros azul 
oscuro. En los pies calza unos zapatos negros de cordón. Utiliza una vestimenta informal ya que el escenario así lo exige. Además, emplea tonos oscuros ya que se trata de ropa de abrigo al grabarse el programa en época de invierno. Sin embargo, debajo de la chaqueta negra e informal lleva una camisa azul clara que le aporta luz al conjunto. Además, al tratarse de una camisa le aporta un toque de formalidad y elegancia.

Desde el primer momento Miguel Ángel Revilla se muestra alegre y tranquilo, haciéndole bromas a Bertín Osborne. Al principio de la entrevista está de pie con las piernas separadas esperando la llegada del presentador. Además, sonríe de forma auténtica durante toda la entrevista, algo que coincide con su actitud.

Llama la atención que en este programa Miguel Ángel Revilla levanta las cejas para enfatizar y expresar sus emociones un total de 15 veces. Con este gesto demuestra la gran expresividad que realiza a través de su rostro.

En cuanto a sus movimientos con las manos realiza un total de 108 gestos. El gesto más repetido es "dedo índice y pulgar unidos y movimiento de arriba abajo", que lo realiza un total de 26 veces durante todo el programa. Este gesto lo emplea para reforzar sus palabras, para acompañar su discurso a través del cual muestra el énfasis y pasión que pone en su discurso.

El segundo movimiento que más realiza con sus manos es el que se denomina "Palma de la mano cerrada con el dedo índice apuntando al frente". Realiza este gesto un total de 16 veces. Al contrario de lo que puede indicar este gesto generalmente (actitud defensiva o acusadora) en este contexto Miguel Ángel Revilla emplea este gesto para puntualizar, para marcar o subrayar las palabras que él mismo considera importantes en su discurso y con el fin último de captar la atención.

El tercer movimiento de manos que más repite es el de juntar la punta de todos los dedos de la mano. Realiza este gesto un total de 13 veces, que emplea con el mismo fin que el gesto que hemos descrito anteriormente, para enfatizar su mensaje.

Llama la atención la cantidad de veces que Miguel Ángel Revilla presenta las manos en los bolsillos de su pantalón ya que emplea este gesto un total de 9 veces. En todas las ocasiones que utiliza este gesto se encuentra caminando de forma lenta y escuchando a Bertín Osborne. En este contexto a través de este gesto demuestra tranquilidad y a la vez estar comodidad y relajación.

Por otro lado, realiza un total de 3 veces el gesto de representación del yo. Ejecutar este gesto 3 veces en un programa que dura 1 hora y 35 minutos no es una cifra elevada. Por lo que no se puede decir que en este programa hable demasiado sobre sí mismo, al contrario de lo que ocurre en el programa que hemos analizado anteriormente.

En cuanto a las piernas, la mayoría del tiempo se presentan cruzadas ya que la entrevista se realiza en un sofá. Al contrario de lo que puede indicar este gesto consideramos por el contexto y las expresiones de su rostro que Miguel Ángel Revilla utiliza lo emplea para estar más cómodo. En ningún caso se emplea con una actitud de defensa. Además, los ratos que se encuentra de pie en ningún momento cruza las piernas, sino que las mantiene separadas.

Por otra parte, el único movimiento que realiza con los pies es un pequeño balanceo que se produce en dos ocasiones. El primer gesto lo realiza al hablar sobre el antiguo nombre que recibía Cantabria que era Provincia de Santander y en segundo lugar cuando Bertín Osborne le pregunta si es cierto que de pequeño quería ser sacerdote. 
En cuanto a sus movimientos en conjunto, Miguel Ángel Revilla emplea movimientos tranquilos compaginados con movimientos firmes y seguros en toda la entrevista.

El timbre que utiliza en esta entrevista es en general medio-alto. En concreto su timbre es más elevado cuando habla sobre el Papa y las posibles medidas que puede llevar a cabo para erradicar el hambre en el mundo. Además, su tono es agudo durante toda la entrevista. También emplea un tempo medio a excepción del minuto 32:18 de programa cuando habla sobre el presidente de Corea del Norte que es más rápido al tratarse de un tema que se nota que le enfada. Con su ritmo pasa lo mismo, mantiene un ritmo medio salvo cuando se pronuncia sobre el Papa y las posibles medidas para erradicar el hambre y cuando se refiere al presidente de Corea del Norte que se convierte en un ritmo agitado.

\subsubsection{Chester in Love}

Chester in Love es un programa de televisión español presentado por Risto Mejide y emitido en la cadena Cuatro. En la actualidad Chester no se encuentra en emisión y el último episodio difundido data de 17 de marzo de 2019. El programa analizado se emitió el 12 de marzo de 2017 a las 21:30 horas y tiene una duración de 33 minutos y 44 segundos. En él, el presentador Risto Mejide y Miguel Ángel Revilla mantienen una charla donde el presidente de Cantabria cuenta anécdotas sobre su vida. Además, interviene la youtuber Soy una Pringada que expone ante Revilla y Risto los problemas a los que se enfrenta su generación.

Este tercer programa analizado corresponde a la entrevista que Miguel Ángel Revilla concedió a Risto Mejide en el año 2017. La entrevista tiene lugar en el plató habitual donde se grava Chester in Love. Es un plató de tamaño medio donde acude público. Cuenta con dos grandes pantallas donde se proyecta en determinados momentos vídeos sobre el invitado. También hay unas letras de gran tamaño en color rojo que rezan "Chester Times". El plató tiene una decoración moderna.

El presidente acude vestido con un traje azul marino, camisa blanca y corbata azul marino. Este traje lo acompaña con unos zapatos negros de cordón. Como complementos lleva en la mano izquierda un reloj de plata y una pulsera de silicona. De esta forma, Miguel Ángel Revilla acude al plató vestido de una forma elegante. Además, elige colores oscuros que contrastan con la camisa blanca que aporta viveza.

Una vez que comienza la entrevista Risto Mejide y Miguel Ángel Revilla se sientan en el sofá típico de las entrevistas en este programa. Miguel Ángel Revilla presenta una postura relajada con la espalda apoyada en el respaldo y las piernas juntas casi la totalidad del tiempo.

Miguel Ángel Revilla sube un total de 11 veces sus cejas. Como hemos comentado, el Presidente de Cantabria es muy expresivo y emplea este gesto frecuentemente, pero sobre todo lo utiliza cuando trata temas personales o que le afectan en primera persona. De estas 11 veces, 3 de ellas realiza este gesto al sorprenderse tras las declaraciones de la youtuber Soy una Pringada. Al Presidente de Cantabria le llama la atención la opinión de esta youtuber sobre su generación ya que dista mucho de la que expresa Revilla.

El movimiento de sus ojos, su mirada, suele mantenerse firme a la del presentador. Además, es una mirada fija que muestra la seguridad que tiene en sí mismo y en su mensaje. Estos gestos se intercalan con una mirada al frente para ver los vídeos que aparecen en la pantalla.

En cuanto a la sonrisa, es auténtica durante toda la entrevista, algo que coincide con su actitud y su rostro. Se aprecia alegría, tranquilidad y seguridad. 
Por otra parte, Miguel Ángel Revilla abre los brazos en 4 ocasiones. En todas ellas emplea este gesto para explicar mejor su mensaje.

En cuanto a sus movimientos con las manos realiza un total de 95 gestos. El más repetido es el denominado en la plantilla "Dedo índice y pulgar unidos y movimiento de arriba abajo". Realiza este gesto 23 veces. En este contexto, Miguel Ángel Revilla utiliza este movimiento para puntualizar y fijar su mensaje, reafirmando sus palabras.

En segundo lugar, mantiene 20 veces la palma de la mano cerrada con el dedo índice apuntando hacia arriba. Este gesto lo emplea con la misma finalidad que el gesto anterior, para enfatizar y precisar sus palabras.

También debemos comentar que en esta entrevista en ningún momento entrelaza las manos ni las introduce en los bolsillos.

Miguel Ángel Revilla emplea un total de 3 veces el gesto de representación del yo en un total de 33 minutos y 44 segundos. Este gesto indica que ha hablado mínimo en 3 ocasiones sobre él.

En cuanto a los gestos realizados con las piernas, al realizarse la entrevista sentados en un sofá la totalidad del tiempo está sentado y con las piernas separadas.

En su conjunto, Miguel Ángel Revilla muestra una actitud segura y a la vez tranquila. Acompaña constantemente su discurso con movimientos que reafirman su mensaje, a la vez muestra una sonrisa auténtica y en ningún momento de la entrevista se le ve incómodo.

Todos estos gestos están acompañados de un timbre medio-alto y un tono agudo ya que es el suyo habitual. En general el tempo que emplea es medio a pesar de que en ciertos temas como cuando habla sobre el presidente de EE.UU. es más rápido o presenta un tempo más lento cuando recuerda a su madre fallecida. Por lo que sus emociones también son expresadas a través del sistema paralingüístico.

\subsubsection{La Sexta Noche}

La Sexta Noche es un programa español emitido en la cadena La Sexta y presentado por Iñaki López, Andrea Ropero y ocasionalmente por Hilario Pino y Paula del Fraile los sábados a las 21:20 horas. En él se tratan temas de actualidad en general a pesar de que el debate político es el núcleo del programa. Además de los presentadores es habitual que acudan representantes políticos o periodistas como colaboradores del programa.

El programa analizado fue emitido el día 11 de mayo de 2019. En este programa se puede ver como a partir del minuto 88 el presentador Iñaki López entrevista a Miguel Ángel Revilla. Es, sin duda, una entrevista diferente ya que tiene lugar en el Parque de la Naturaleza de Cabárceno. Allí, Miguel Ángel Revilla concede su primera entrevista después de las elecciones generales de 2019.

Miguel Ángel Revilla asiste a la entrevista vestido con una camisa blanca con rayas finas y pantalón de vestir gris oscuro acompañado por un cinturón en color negro. En los pies calza zapatos negros con cordón. En conjunto, presenta un aspecto más desenfadado del habitual, pero no pierde la formalidad ya que se decanta por una camisa. Además, el Presidente de Cantabria opta por una camisa de color blanca, esta elección se puede deber a que como se aprecia en el vídeo hacía buen tiempo. 
Durante la entrevista, Miguel Ángel Revilla muestra una postura relajada. A pesar de que en esta ocasión está de pie, su rostro y movimientos denotan una actitud de calma. En ningún momento se pone nervioso. Además de estar tranquilo se nota que está seguro de sí mismo, así lo afirman los gestos de los que vamos a hablar más adelante.

La mirada del Presidente de Cantabria se mantiene firme al presentador Iñaki López, mirándole a los ojos. Además, es una mirada estable que expresa seguridad y calma.

Durante toda la entrevista muestra una sonrisa auténtica. En concreto en el minuto 1:26:26 de programa le comenta al presentador que quiere hacer autopromoción sobre su candidatura en $\mathrm{La}$ Sexta Noche. Esta declaración le hace gracia al propio protagonista que muestra una amplia sonrisa.

Durante el programa, solo realiza un único levantamiento de cejas. Efectúa este gesto al narrar su disconformidad por el tratamiento que recibe el clima de Cantabria en los medios de comunicación.

Además, en esta entrevista si muestra una actitud de enfado, concretamente en dos ocasiones. La primera cuando comenta que tiene una "guerra" con los meteorólogos ya que siempre prevén lluvia para Cantabria y la segunda ocasión al explicar que en las encuestas no se predecía que el Partido Regionalista de Cantabria consiguiera un diputado en el Congreso.

En cuanto a sus movimientos con las manos, realiza un total de 52 gestos. El más repetido es el que ejecuta cuando coloca la palma de la mano cerrada y el dedo índice apuntando hacia arriba ya que repite este gesto 11 veces. En este contexto considero que realiza este gesto para reforzar su mensaje y enfatizar las palabras importantes. Con este mismo fin también realiza 6 veces el gesto de juntar la punta de todos los dedos de la mano.

Miguel Ángel Revilla entrelaza las manos 4 veces. Llama la atención que no realiza este gesto ante una idéntica temática, sino que lo hace ante temas diversos. Por lo que pienso que el significado de este gesto viene a indicar calma y tranquilidad.

En cuanto a los movimientos de sus piernas al tratarse de una entrevista que se realiza de pie, Miguel Ángel Revilla se desplaza caminando lentamente mientras charla con el presentador. En diversas ocasiones se para para profundizar y recalcar el tema sobre el que está hablando.

En cuanto al sistema paralingüístico, el Presidente de Cantabria presenta un timbre medio-alto durante toda la entrevista, tono agudo, tempo o velocidad medio y un ritmo suave.

\subsection{Comparación de resultados de los cuatro programas analizados: Espejo Público, Mi casa es la tuya, Chester in Love y La Sexta Noche}

Es complicado realizar una comparación de los resultados obtenidos. La dificultad recae en que cada programa tiene una duración diferente. De esta forma, en el programa de mayor duración (Mi casa es la tuya con 1 hora 33 minutos y 44 segundos) el protagonista realiza un número superior de gestos que en el programa de menor duración (La Sexta Noche con 20 minutos y 19 segundos) como es lógico.

De todas formas, vamos a realizar esta comparación teniendo en cuenta las características de cada programa.

En primer lugar, debemos apuntar que en Chester in Love es donde más tiempo Miguel Ángel Revilla está sentado. Esto se debe a que la entrevista se lleva a cabo en un sofá. 
Por otro lado, el programa en el que más tiempo se encuentra de pie es en La Sexta Noche puesto que se produce en los alrededores del Parque de la Naturaleza de Cabárceno.

Espejo Público y Chester in Love son los programas en los que hace un mayor número de asentimientos. Emplea este gesto 2 veces en cada programa. Por el contrario, en Espejo Público es donde más niega con la cabeza, ejecuta este movimiento un total de 4 veces.

Por otro lado, en el programa Mi casa es la tuya Miguel Ángel Revilla sube la ceja un total de 15 veces, y es el programa en el que más realiza este gesto. Le sigue el programa Chester in Love con 11 veces, Espejo Público con 3 y finalmente La Sexta Noche con 1.

En cuanto a las emociones del rostro, el Presidente de Cantabria muestra alegría y una sonrisa auténtica en los cuatro programas. Sin embargo, en La Sexta Noche muestra síntomas de enfado en 2 ocasiones y en Chester in Love se puede percibir melancolía en su rostro de nuevo en dos ocasiones.

En cuanto a los gestos de las manos tanto en Mi casa es la tuya como en Chester in Love, el gesto más repetido es el denominado "Dedo índice y pulgar unidos y movimiento de arriba abajo". Además, se reproduce un número de veces muy similar ya que en el primer programa se realiza 26 veces y en el segundo 23.

En cuanto a la postura de sus piernas, en Espejo Público y Mi casa es la tuya Miguel Ángel está sentado en un sofá con las piernas cruzadas la mayoría del tiempo. Sin embargo, en Chester in Love a pesar de que casi la totalidad del tiempo está sentado, mantiene las piernas juntas, pero no cruzadas. Finalmente, sólo en el programa La Sexta Noche está de pie y con las piernas separadas, nunca cruzadas.

En el caso del sistema paralingüístico, Miguel Ángel Revilla emplea en los cuatro programas un timbre medio-alto, tono agudo, tempo medio y ritmo suave. Sin embargo, en el programa Chester in Love es donde más varía su paralingüística debido a los temas que se tratan en la entrevista. Modifica su tempo que pasa de medio a suave al recordar a su madre fallecida. También modifica su tempo a uno más rápido al hablar sobre el presidente de Estados Unidos, Donald Trump.

En el caso de la indumentaria de Miguel Ángel Revilla, podemos afirmar que el protagonista de éste trabajo se viste con ropa formal en dos de los programas analizados (Espejo Público y Chester in Love). Pasa lo contrario en Mi casa es la tuya y La Sexta Noche que acude con ropa más desenfadada. La elección de una vestimenta más o menos formal puede deberse al escenario donde transcurre la entrevista. En los dos programas que son grabados en su tierra, Cantabria, Miguel Ángel Revilla opta por una vestimenta informal, mostrando su lado más campechano y cercano a sus paisanos. Sin embargo, en los platós de televisión de grandes ciudades (Madrid y Barcelona) se presenta con traje y corbata, mostrando a través de su vestimenta la importancia de su cargo. También debemos apuntar que las características del escenario así lo "exigen”, ya que por ejemplo llamaría la atención que Miguel Ángel Revilla vistiera traje y corbata en un mirador de los Picos de Europa que es una de las localizaciones del programa Mi casa es la tuya.

\section{Conclusiones}

Tras la realización del análisis de la comunicación no verbal de Miguel Ángel Revilla a través de la plantilla llevada a cabo ad hoc para este trabajo, se ha llegado a la obtención de unas conclusiones. Éstas se encuentran directamente relacionadas con los objetivos e hipótesis fijados al principio de la investigación. 
Podemos afirmar que:

1. En cuanto al primer objetivo planteado al inicio de la investigación, donde se pretende averiguar cuál es el gesto más repetido por Miguel Ángel Revilla en los cuatro programas analizados, podemos afirmar que se trata del denominado en la plantilla "Dedos índice y pulgar unidos y movimiento de arriba abajo" realizado un total de 60 veces.

2. Con el segundo objetivo se busca descubrir qué transmite el gesto más repetido por el Presidente de Cantabria en los cuatro programas de infoentretenimiento televisivos elegidos. Tras el análisis de los programas se puede concluir que este gesto transmite firmeza, convencimiento y confianza en su mensaje. Además, lo emplea para reafirmar sus palabras.

3. El objetivo número 3, con el que se trata de comprobar si los gestos de Miguel Ángel Revilla se corresponden con un discurso creíble, queda afirmado ya que a través de los resultados que hemos obtenido se puede comprobar que los gestos más repetidos son aquellos que se corresponden con la transmisión de credibilidad. Por ejemplo, la función básica del segundo gesto más repetido (palma de la mano hacia arriba) que Miguel Ángel Revilla realiza un total de 32 veces, se corresponde con la transmisión de un mensaje verosímil.

4. Con el objetivo número 4 se pretende comparar la comunicación no verbal de Miguel Ángel Revilla en los programas de infoentretenimiento televisivos del año 2017 al 2019. Tras la realización de este trabajo se puede afirmar que en su conjunto, los gestos empleados por Miguel Ángel Revilla en los programas analizados pertenecientes al año 2017 son más inseguros que los que lleva a cabo en los dos programas de infoentretenimiento del 2019, año en el que el Presidente de Cantabria consigue ganar las elecciones con mayoría de votos, hecho que no ocurrió en el 2017. Además, en los programas del 2019 su rostro muestra más euforia y alegría.

5. En cuanto al único objetivo secundario que se plantea en este trabajo, que trata de detectar si existe una influencia del entorno en la indumentaria que utiliza Miguel Ángel Revilla durante los cuatro programas seleccionados, podemos concluir que Miguel Ángel Revilla emplea un estilo informal en los dos programas rodados en Cantabria. Sin embargo, no sucede lo mismo en los dos programas grabados en grandes ciudades (Madrid y Barcelona) que se presenta con un estilo formal, con traje y corbata. Además, cuando aparece vestido de forma informal su papel es la de ser anfitrión. En los dos programas donde viste traje y corbata acude para ser entrevistado como Presidente de Cantabria. La indumentaria de Miguel Ángel Revilla se modifica dependiendo del rol social que desempeñe.

A partir de las hipótesis planteadas para el desarrollo de este trabajo y mediante los resultados obtenidos tras realizar el análisis se ha llegado a las siguientes conclusiones que pasan a comprobar o refutar dichos supuestos:

- Hipótesis I: El Presidente de Cantabria, a través de su comunicación no verbal, se muestra seguro de sí mismo en sus intervenciones en programas de infoentretenimiento televisivos.

Esta hipótesis queda afirmada ya que Miguel Ángel Revilla, tal y como se explica en el objetivo número 2 de este trabajo y a través de los resultados que hemos obtenido al realizar el análisis, utiliza una gran cantidad de gestos que transmiten seguridad en su discurso. Por ejemplo, su postura en todos los programas es siempre erguida, su mirada directa y firme a los ojos del presentador. Realiza un total de 11 veces el gesto denominado en la plantilla "Juntar la punta de los dedos de la mano derecha con los de la izquierda con el mismo dedo correspondiente y separando la palma" con el que indica seguridad en sí mismo y en su mensaje. Además, mueve poco los pies, algo que también transmite confianza y seguridad.

- Hipótesis II: En las entrevistas concedidas después de ganar las elecciones autonómicas de este mismo año, Miguel Ángel Revilla muestra a través de sus gestos más seguridad y alegría que en las intervenciones del 2019 donde ya ha ganado por mayoría las elecciones autonómicas y conseguido un diputado en las elecciones generales. 
Como se explica en las conclusiones del objetivo número 4, esta hipótesis queda afirmada. Miguel Ángel Revilla, como se puede comprobar en las plantillas y en los resultados obtenidos del análisis, muestra en su rostro más señales de alegría y una sonrisa auténtica en las intervenciones del año 2019. Además, su tono de voz es más elevado y realiza un número mayor de gestos. También se produce un aumento de los gestos que transmiten seguridad.

- Hipótesis III: Miguel Ángel Revilla emplea una vestimenta más informal cuando se encuentra en su tierra, Cantabria, hecho que le aporta una imagen más cercana y campechana.

Esta afirmación también queda confirmada. Como se puede ver en los programas analizados, Miguel Ángel Revilla utiliza una vestimenta informal únicamente cuando se encuentra en Cantabria. Además de la vestimenta, a través de su discurso relata su pasión por el campo y en reiteradas ocasiones narra la vida humilde que llevó en su pueblo natal cuando era niño. Por lo que la vestimenta informal y este discurso le aportan una imagen más cercana y campechana.

- Hipótesis IV: El Presidente de Cantabria no pone ningún inconveniente en contestar preguntas sobre cuestiones de su vida privada.

Como se puede comprobar en la tercera columna de las plantillas donde se realizan el análisis de la comunicación no verbal de Miguel Ángel Revilla, el Presidente de Cantabria habla sobre su infancia, su mujer, sus hijas e incluso sobre su tristeza al enterarse del fallecimiento de su madre. Por lo que esta hipótesis queda confirmada.

\section{Referencias bibliográficas}

AIMC (2019). Marco General de los medios en España 2019. https://www.aimc.es/a1mcc0nt3nt/uploads/2019/01/marco19.pdf

Alonso González, M. (2017). Infoentretenimiento y política: el caso de las elecciones de 2015 y 2016 en España. Revista de Comunicación de la SEECI, (44), 87-113.

file://D:/Documentos/Downloads/DialnetInfoentretenimientoYPoliticaElCasoDeL asEleccionesD-6295963\%20(3).pdf

Alonso Ondiviela, A. (2017). El papel de la comunicación no verbal en la producción y en la recepción del discurso del intérprete. Trabajo de fin de grado, Universidad de Valladolid, Soria. https://uvadoc.uva.es/bitstream/handle/10324/28690/TFG-

$\mathrm{O} \%$ 201143.pdf? sequence $=1 \&$ isAllowed $=\mathrm{y}$

Antena 3 (2017, noviembre 10). Miguel Ángel Revilla canta en directo el nuevo himno que propone para Cantabria. https://www.antena3.com/programas/el-hormiguero/invitados/miguel-angelrevilla-canta-en-directo-el-nuevo-himno-que-proponepara\%20cantabria_2016092757eaea1c0cf26658235e9d75.html

Barlovento Comunicación (2009). Informe de audiencia debate electoral 23 abril de 2019. https://www.barloventocomunicacion.es/wp- content/uploads/2019/04/NOTA-DE-PRENSADEBATE-23.04.19-3.pdf

Berrocal Gonzalo, S. (2015). Politainment: el gran espectáculo político televisivo. https://compolitica.com/politainment-el-gran-espectaculo-politico-televisivo/

Berrocal Gonzalo, S. y Cebrián Guinovart, E. (2009). El «infoentretenimiento» político televisivo. Un análisis de las primeras intervenciones de Zapatero y Rajoy en «Tengo una pregunta para usted». Textual y Visua Media, (2), 41-60.

http://textualvisualmedia.com/index.php/txtvmedia/article/view/27/23 
Berrocal Gonzalo, S., Campos Domínguez, E. y Redondo García, M. (2012). El "infoentretenimiento" político en Internet: La presencia de cinco líderes europeos en YouTube. RIPS: Revista de Investigaciones políticas y sociológicas, 11(4), 107-131.

http://www.redalyc.org/pdf/380/38028440007.pdf

Berrocal Gonzalo, S., Redondo García, M., Martín Jiménez, V. y Campos Domínguez, E. (2014). La presencia del infoentretenimiento en los canales generalistas de la TDT española. Revista Latina de Comunicación Social, (69), 66-103.

http://www.revistalatinacs.org/069/paper/1002_UVa/RLCS_paper1002.pdf

Caamaño, A. (2016). El infoentretenimiento político televisivo. Un análisis de las estrategias comunicativas utilizadas por los principales líderes políticos en precampaña electoral en El Programa de Ana Rosa. Trabajo fin de grado, Universidad de Valladolid, Soria. https://uvadoc.uva.es/bitstream/10324/19546/1/TFG_F_2016_239.pdf

Cabana, G. (2008). ¡Cuidado! Tus gestos te traicionan. Sirio.

Canel, M. J. (2005). Comunicación política: Técnicas y estrategias para la sociedad de la información. Tecnos.

Carabajal, J. D. (2011). Los nuevos aires en la comunicación política: la TV y la política, una alianza a favor del protagonismo personal. Inferencia política, 2, 1-8.

Cartes Barroso, M. J. (2018). El uso de Instagram por los partidos políticos catalanes durante el referéndum del 1-0. Revista de Comunicación de la SEECI, (47), 17-36.

http://www.seeci.net/revista/index.php/seeci/article/view/517/pdf_280

Cestero Mancera, A. M. (2006). La comunicación no verbal y el estudio de su incidencia en fenómenos discursivos como la ironía. ELUA, (20), 57-77.

https://rua.ua.es/dspace/bitstream/10045/6074/1/ELUA_20_03.pdf

Davis, F. (2010). La comunicación no verbal. FGS.

https://comunicacionenlaunsj.files.wordpress.com/2011/09/la-comunicacic3b3n-no-verbal.pdf

EuropaPress (2019, abril 11). Revilla, el presidente autonómico con mejor índice de transparencia, según Dyntra. EuropaPress. https://www.europapress.es/cantabria/noticia-revilla-presidenteautonomico-mejor-indice-transparencia-dyntra-20190411143300.html

Forner, A. (1987). La comunicación no verbal. Grao.

Gallego Reguera, M. y Bernárdez Rodal, A. (2017). Influencia y repercusión mediática de los debates "cara a cara" celebrados ante las elecciones generales de 2008 en España: José Luis Rodríguez Zapatero (PSOE) vs. Mariano Rajoy (PP). Vivat Academia. Revista de Comunicación, (141), 139-154. http://doi.org/10.15178/va.2017.141.139-154

García Avilés, J. A. (2007). El infoentretenimiento en los informativos líderes de audiencia en la Unión Europea. Análisis: cuadernos de comunicación y cultura, (35), 47-63. http://dspace.umh.es/bitstream/11000/4557/1/22infoentretenimiento $\% 20$ garcia $\% 20$ Avil\%C3\%A9s.pdf 
García, L. (2018, noviembre 10). El PRC cumple 40 años con el objetivo de llevar a Revilla a su primera victoria. Eldiario.es. https://www.eldiario.es/norte/cantabria/politica/PRC-objetivo-Revilla-victoriaelectoral_0_834316865.html

Garrido, C. (2019, febrero 25). Por qué no deberías beber leche cruda como Miguel Ángel Revilla. $A B C$. https://www.abc.es/salud/enfermedades/abci-no- deberias-beber-leche-cruda-como-miguelangel-revilla-201902251214_noticia.html

Hall, E. T. (1973). La dimensión oculta. Enfoque antropológico del uso del espacio. Nuevo Urbanismo.

Kantar Media (2019). Medición de televisión y vídeo en plataformas y dispositivos. https://www.kantarmedia.com/es/soluciones/medicion-de-audiencias/television- y-video

Laguna, A. (2011). Liderazgo y Comunicación: la personalización de la política. Anàlisi: Quaderns de comunicación $i$ cultura, (43), 45-57.

https://ruidera.uclm.es/xmlui/bitstream/handle/10578/1786/fi_1322582592-

131124831pb1.pdf? sequence $=1 \&$ isAllowed $=\mathrm{y}$

Laguna, E. (2012). Globalización, Civilización, Americanización: cabos sueltos. Historiografias: revista de historia y teoría, (3), 8-25. file://D:/Documentos/Downloads/DialnetGlobalizacionCivilizacionAmericanizacion-4040109\%20(1).pdf

Mancini, P. (1995). Americanización y modernización. Breve historia de la campaña electoral. En A. Muñoz-Alonso y J. I. Rospir (eds.), Comunicación política (pp. 141-168). Universitas.

Martínez Pandiani, G. (2006). El impacto de la televisión en la Comunicación Política moderna. Signos Universitarios: Revista de la universidad del Salvador, 25(Extra 1), 67-88. file://D:/Documentos/Downloads/2955- 10053-1-PB\%20(1).pdf

Monzón, C. (2006). Opinión pública, comunicación y política. Tecnos.

Moyá Ruíz, M. T. (2016). Habilidades comunicativas y comunicación política. Tesis doctoral, Universidad Miguel Hernández, Elche (Alicante).

http://dspace.umh.es/bitstream/11000/3208/1/TD\%20Moya\%20Ruiz\%2C\%20Maria\%20Teresa.pdf

Oliva, L. y Sitjà, X. (2007). Las noticias en radio y televisión: Periodismo audiovisual y televisión. Omega S.A.

Orejuela, S. (2006). Proceso de globalización de las campañas electorales: de la americanización a la profesionalización. Revista de comunicación, (5), 55-87.

file://D:/Documentos/Downloads/Dialnet- ProcesoDeGlobalizacionDeLasCampanasElectorales$3870684 \% 20(2) \cdot p d f$

Ortells Badenes, S. (2009). La mercantilización de la información: la nueva era informativa en televisión. Revista Latina de Comunicación Social, 12(64), 341-353.

http://www.revistalatinacs.org/09/art/28_827_46_ULEPICC_07/RLCS_art827.pdf

Ortells Badenes, S. (2011). La consolidación de los programas de infoentretenimiento en el panorama televisivo español. Fórum de Recerca, (16), 279-292.

http://repositori.uji.es/xmlui/bitstream/handle/10234/77227/fr_2011_21.pdf?sequence=1\&is

Allowed $=\mathrm{y}$ 
Ortells Badenes, S. (2012). Infoentretenimiento y periodismo político en televisión. En A. CaseroRipollés (ed.), Periodismo político en España: concepciones, tensiones y elecciones (pp.101-117). Sociedad Latina de Comunicación Social.

http://repositori.uji.es/xmlui/bitstream/handle/10234/73587/CAL33Casero.pdf? sequence=6

Padilla Castillo, G. (2015). La espectacularización del debate electoral: estudio del caso en estados unidos. Vivat Academia. Revista de Comunicación, (132), 162-181.

http://www.vivatacademia.net/index.php/vivat/article/view/587/130

Padilla Castillo, G. (2019). [Reseña del libro La Comunicación de los partidos políticos en España tras el bipartidismo, de E. Aladro Vico y P. Requeijo Rey (Coords.)]. Vivat Academia. Revista de Comunicación, (146), 161-162. http://doi.org/10.15178/va.2019.146.161-162

Pease, A. (2011). El arte de negociar y persuadir. Amat.

Pease, A. y Pease, B. (2006). El lenguaje del cuerpo: Cómo interpretar a los demás a través de sus gestos. Amat Editorial.

https://books.google.es/books?hl=es\&lr=\&id=6uQXHfdJBI4C\&oi=fnd\&pg=PA15\&dq=libro+pea $\mathrm{se}+$ comunicacion + no + verbal\&ots $=\mathrm{j} 2 \mathrm{~s} X \mathrm{TycKq} 8 \&$ sig $=$ huqjldBG9sU-

$\mathrm{kWm} 24 \mathrm{EzM} 51 \mathrm{Jk} 5 \mathrm{u} 0 \# \mathrm{v}=$ onepage \&q\&f$=$ false

Pérez, D. (2003). Técnica de comunicación politica: el lenguaje de los partidos. Tecnos.

Poyatos, F. (1994). La comunicación no verbal. Cultura, lenguaje y conversación. Istmo.

Poyatos, F. (2003). La comunicación no verbal: algunas de sus perspectivas de estudio e https://revistas.um.es/ril/article/view/5741

Rodríguez Vidales, Y. y Padilla Castillo, G. (2018). El cine político puro: la fícción como inspiración/reflejo de la espectacularización política. Revista de Comunicación de la SEECI, (45), 15-42. http://www.seeci.net/revista/index.php/seeci/article/view/506/pdf_243

Sartori, G. (2003). Homo videns: La sociedad teledirigida. Taurus.

Starck, S. D. (1997). Local News: The Biggest Scandal on TV. It's shallow, it's stupit, it misleads the public - It's the local news. The Washington Monthy, 29(6), 39.

Valencia, A. (2015). Políticas e imagen en una democracia de audiencia. Paradigma: Revista Universitaria de Cultura, (18), 27-29.

https://riuma.uma.es/xmlui/bitstream/handle/10630/8814/Valencia.pdf?sequenc e=1

\section{AUTORES:}

\section{Noelia Fontecoba}

Noelia Fontecoba es Graduada en Publicidad y Relaciones Públicas por la Universidad de Vigo (Facultad de Ciencias Sociales y de la Comunicación, Campus de Pontevedra) y autora principal de este trabajo, derivado de la investigación realizada en el marco de su trabajo de fin de grado.

noeliafontecoba@gmail.com 


\section{Ana Belén Fernández-Souto}

Licenciada y Doctora en Publicidad y Relaciones Públicas por la Universidad de Vigo, de la que es profesora titular. Ha realizado estancias de docencia en diversas universidades internacionales (U. Dámaso Alonso Larrañaga, Uruguay; U. Minas Gerais, Brasil; U. Costa Rica, Costa Rica; U. Dubrovnik, Croacia; U Autónoma de Querétaro, México; U. Nacional Autónoma de México, México; U. Católica del Norte, Chile; Lumsa Universitá, Italia; Universidad Autónoma, México; U. Fernando Pessoa, Portugal o Universidade de Minho, Portugal, entre otras) y ha publicado varios libros y artículos de carácter científico centrados fundamentalmente en el ámbito de las relaciones públicas, el protocolo, la imagen de marca y la comunicación de crisis y es, además, coordinadora del grupo de investigación "CP2: Comunicación Persuasiva" de la UVigo. Su índice i10, 3 (abril 2018).

abfsouto@uvigo.es

Índice H: 5

Orcid ID: https://orcid.org/0000-0003-2685-0604

Google Scholar: $\underline{\text { https://scholar.google.es/citations?user=6UiwqukAAAAJ\&hl=es }}$

\section{Iván Puentes-Rivera}

Doctor en Comunicación, Licenciado en Publicidad y Relaciones Públicas y Máster en Investigación en Comunicación por la Universidad de Vigo, es profesor de comunicación en el Departamento de Sociología y Ciencias de la Comunicación de la Universidad de A Coruña (España) y miembro del grupo de investigación CP2: Comunicación Persuasiva de la Universidad de Vigo. Anteriormente, fue profesor de las facultades de Ciencias de la Comunicación de esa misma universidad y de la Universidad de Santiago de Compostela, en la que también trabajó como técnico superior de apoyo a la investigación. Vinculado, como Secretario Técnico, a la Red Internacional de Investigación de la Gestión de la Comunicación (XESCOM), es autor de diferentes artículos y trabajos sobre gestión de la comunicación, especialmente en los campos de la comunicación política, electoral e institucional y de los debates electorales en televisión. Ha realizado estancias de docencia e investigación en diversas universidades portuguesas y ecuatorianas y posee una variada experiencia profesional en el ámbito de la gestión y la comunicación política. Su índice H es 7 y el i10, 3 (noviembre de 2019). i.puentes@udc.es

Índice H: 7

Orcid ID: http://orcid.org/0000-0003-1982-0984

Google Scholar: https://scholar.google.es/citations?user=6pZK8ecAAAAJ\&hl=es 